\title{
An Association Study between Genetic Polymorphism in the Interleukin-6 Receptor Gene and Coronary Heart Disease
}

\author{
Jiangqing Zhou, ${ }^{1}$ Xiaoliang Chen, ${ }^{1,2}$ Huadan Ye, ${ }^{2}$ Ping Peng, ${ }^{1,2}$ Yanna Ba, \\ Xi Yang, ${ }^{1}$ Xiaoyan Huang, ${ }^{1}$ Yae Lu, ${ }^{1}$ Xin Jiang, ${ }^{1}$ Jiangfang Lian, ${ }^{1}$ and Shiwei Duan ${ }^{2}$ \\ ${ }^{1}$ Ningbo Medical Center, Lihuili Hospital, Ningbo University, 57 Xingning Road, Ningbo, Zhejiang 315211, China \\ ${ }^{2}$ School of Medicine, Ningbo University, 818 Fenghua Road, Ningbo, Zhejiang 315211, China \\ Correspondence should be addressed to Jiangfang Lian; hjmpin@163.com and Shiwei Duan; duanshiwei@nbu.edu.cn
}

Received 7 February 2014; Revised 9 April 2014; Accepted 16 April 2014; Published 26 May 2014

Academic Editor: Hongwei Wang

Copyright (C) 2014 Jiangqing Zhou et al. This is an open access article distributed under the Creative Commons Attribution License, which permits unrestricted use, distribution, and reproduction in any medium, provided the original work is properly cited.

The goal of our study is to test the association of IL6R rs7529229 polymorphism with CHD through a case-control study in Han Chinese population and a meta-analysis. Our result showed there is a lack of association between IL6R rs7529229 polymorphism and CHD on both genotype and allele levels in Han Chinese $(P>0.05)$. However, a meta-analysis among 11678 cases and 12861 controls showed that rs7529229-C allele was significantly associated with a decreased risk of CHD, especially in Europeans $(P<$ 0.0001 , odds ratio $=0.93,95 \%$ confidential interval $=0.89-0.96$ ). Since there is significant difference among different populations, further studies are warranted to test the contribution of rs7529229 to CHD in other ethnic populations.

\section{Introduction}

Coronary heart disease (CHD) is one of the leading causes of human deaths in the developed and developing countries such as China [1]. As a complex disease, $\mathrm{CHD}$ results from the interaction between genetic and environmental factors. CHD is one of the most common manifestations of atherosclerosis that is related to inflammation [2]. CHD is regarded as a chronic inflammatory disease [3] that has been shown to be associated with the response to inflammatory signaling [4].

Interleukin-6 is an inflammatory cytokine [5], whose synthesis is stimulated by its binding to IL6R. IL6R signaling activates an intracellular signaling cascade leading to the inflammatory response [6] and thus has become an important therapeutic target for prevention of $\mathrm{CHD}[7,8]$.

Human IL6R is located on 1q21, a susceptible locus for CHD. IL6R rs7529229 is a T/C variation associated with both IL6R level and a decreased risk of CHD events in Europeans [7]. Since there is a lack of evidence concerning its role in CHD in Han Chinese, the goal of our study was to replicate the association between IL6R rs7529229 polymorphism and CHD in Han Chinese. In addition, we performed a meta-analysis of the available case-control studies between rs7529229 of IL6R gene and CHD.

\section{Methods}

2.1. Sample Collection. A total of 459 unrelated individuals were selected between May 2011 and November 2013 from Ningbo Lihuili Hospital, Zhejiang, China. Of these, 263 patients had CHD (males: 181; females: 82; age: $61.04 \pm 8.68$ years) and 196 patients were non-CHD controls (males: 98; females: 98 ; age: $57.76 \pm 7.97$ years). The patients had been examined by standardized coronary angiography according to the Seldinger method [9] and were judged by at least two independent cardiologists. In CHD cases, patients $(n=$ 263) were diagnosed with the angiographic evidence that coronary artery stenosis was greater than $50 \%$ in one or more major coronary arteries [10]. Gensini scoring system was used to determine the severity of CHD [11]. A total of 196 patients, who did not have detectable coronary stenosis and atherosclerotic vascular disease, were considered as controls. All individuals had no cardiomyopathy or congenital heart, liver, or renal diseases. All the samples were Han Chinese living in Ningbo of China. The blood samples were collected by the same investigators. Blood samples were collected in $3.2 \%$ citrate sodium-treated tubes and then stored at $-80^{\circ} \mathrm{C}$. The study protocol was approved by the Ethics Committee of 
Lihuili Hospital in Ningbo and informed written consent was obtained from all subjects.

2.2. PCR Amplification and SNP Genotyping. Human genomic DNA was prepared from peripheral blood samples using the nucleic acid extraction automatic analyzer (LabAid 820, Xiamen, China) and was quantified using the PicoGreen dsDNA Quantification kit (Molecular Probes Inc., Eugene, OR, USA). Amplification was performed on the ABI GeneAmp PCR System 9700 Dual 96-Well Sample Block Module (Applied Biosystems, Foster City, CA, USA). Genomic DNA was subjected to polymerase chain reac-tion (PCR) with primers specific to IL6R gene. The sequences of the two allele-specific primers were $5^{\prime}$-GCGGCAGGGCGGCAATGTGGTCGTGGTGAGTTACC C-3' and $5^{\prime}$-GATTACCGAATGTGGTCGTGGTGAGTTA CCT-3'. The sequence of a reverse primer was 5 -TTTCTATGATTCCCTTTCACAGAGGTTTGA- $3^{\prime}$. The reaction was performed with an initial denaturation stage at $95^{\circ} \mathrm{C}$ for $30 \mathrm{sec}$, followed by 40 cycles at $95^{\circ} \mathrm{C}$ for $30 \mathrm{sec}, 59^{\circ} \mathrm{C}$ for $30 \mathrm{sec}$, and $72^{\circ} \mathrm{C}$ for $30 \mathrm{sec}$, and a final extension at $72^{\circ} \mathrm{C}$ for $30 \mathrm{sec}$. Genotyping of the PCR products was performed on the Roche LightCycler 480 Fluorescence Real-Time PCR System (Roche, Rotkreuz, Switzerland) using melting temperature shift (Tm-shift) according to the manufacturer's instructions $[12,13]$. Tm-shift method uses two allele-specific primers and one reverse primer to amplify the polymorphic region encoding the targeted variant, and genotypes can be determined by inspection of a melting curve [14, 15] (Figure 1). To verify the repeatability and stability of experiment, $5 \%$ of random samples and 18 control samples (including 9 negative and 9 positive controls) were used for quality control.

2.3. Retrieval of Published Studies. A search was performed for the publications from 2008 to 2013 in the electronic databases (including PubMed, EMbase, Web of Science, and Cochrane Library). The search keywords included "coronary heart disease" or "coronary artery disease" or "myocardial infarction" combined with "IL6R" or "interleukin-6 receptor" or "rs7529229" or "polymorphism" and "genetic association." We read the full-text articles to collect the relevant information. References listed on the retrieved articles and previous meta-analyses on this subject were searched to appraise other studies of potential relevance. The included studies for the meta-analysis need to be case-control design and need to have information consisting of ORs and their 95\% CIs or genotyping data to measure the relative risk. Data extraction was carried out by at least two reviewers (Xiaoliang Chen and Ping Peng) on a standard protocol, and the consensus data were established by discussion. In the meta-analyses, the following data collection was included: name of the first author, publication year, country, ethnic population, study stage, numbers of individuals in the case and the control groups, OR, and 95\% CI.

2.4. Statistical Analysis. Hardy-Weinberg equilibrium (HWE) was analyzed using the Arlequin software (v3.5)

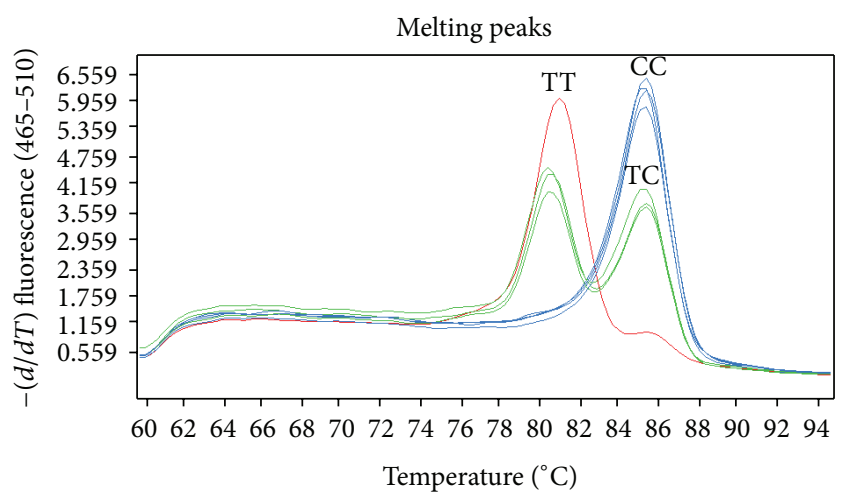

Figure 1: Melting temperature- (Tm-) shift method was used for SNP genotyping.

[16]. The statistical software package SPSS v.18.0 (SPSS, Chicago, IL, USA) was used for the following analyses. Continuous data were expressed as mean \pm SD and Student $t$-test was employed to analyze differences between two study groups. $\chi^{2}$ analysis was used to compare the categorical variables. Genotype and allele frequencies between CHD cases and healthy controls among the different subgroups were compared using $\chi^{2}$ test. Association between Gensini scores and rs7529229 was compared using linear regression test. Mann-Whitney test was used for the association of Gensini scores with rs7529229 under the dominant and the recessive models. The power of the study was estimated by the Power and Sample Size Calculation software (v3.0.43) [17]. Meta-analysis was performed by Stata software version 11.0 (Stata Corporation, College Station, TX) and in accordance with Stroup's study [18]. Heterogeneity of the studies was evaluated by the $I^{2}$ statistic at the significant level of 0.05 . The combined odds ratios (ORs) along with their $95 \%$ confidence intervals (CIs) were assessed with inverse-variance fixedeffect model (subtotal $I^{2}=20.4 \%, P=0.274$; overall $I^{2}=$ 12.5\%, $P=0.441$ ) [19]. Subgroup meta-analysis was performed by ethnicity. Sensitivity analysis was conducted by omitting each study in turn. Funnel plots and Egger regression tests [20] were used to estimate the publication bias. A two-sided $P<0.05$ was considered to be statistically significant.

\section{Results}

3.1. Basic Characteristics of the Study Population. As shown in Table 1, the prevalence of essential hypertension (EH), diabetes mellitus (DM), and smoking history was significantly higher in CHD patients than controls $(P<0.05)$. However, there were no significant differences between the two groups for a series of biochemical parameters, including high-density lipoprotein cholesterol (HDL-C), triglycerides, low-density lipoprotein cholesterol (LDL-C), and total cholesterol.

3.2. Genotype and Allele Distribution of rs7529229 in CHD Cases and Controls. Genotype distribution of rs7529229 in both CHD cases and controls met HWE (Table 2). Genotype 
TABLE 1: Basic characteristics of the study population.

\begin{tabular}{lccc}
\hline & CHD $(n=263)$ & Controls $(n=196)$ & $P$ \\
\hline Male, $n(\%)$ & $182(69.2 \%)$ & $98(50 \%)$ & $<0.001$ \\
Smoking, $n(\%)$ & $111(43.5 \%)$ & $53(27.3 \%)$ & $<0.001$ \\
Hypertension, $n(\%)$ & $161(63.1 \%)$ & $100(51.5 \%)$ & 0.014 \\
Diabetes, $n$ (\%) & $53(20.7 \%)$ & $12(6.1 \%)$ & $<0.001$ \\
Mean age, years & $61.04 \pm 8.68$ & $57.76 \pm 7.97$ & $<0.001$ \\
LDL-C (mmol/L) & $2.54 \pm 0.94$ & $2.49 \pm 0.89$ & 0.600 \\
Total cholesterol (mmol/L) & $4.33 \pm 1.10$ & $4.28 \pm 1.01$ & 0.620 \\
HDL-C (mmol/L) & $1.06 \pm 0.30$ & $1.12 \pm 0.29$ & 0.048 \\
Triglycerides (mmol/L) & $1.64 \pm 1.06$ & $1.50 \pm 0.86$ & 0.125 \\
\hline
\end{tabular}

TABLE 2: Genotype and allele distribution of rs7529229 in CHD cases and controls.

\begin{tabular}{|c|c|c|c|c|c|c|c|c|}
\hline Group & Genotype (TT/TC/CC) & $\chi^{2}$ & $P(\mathrm{df}=2)$ & HWE & Allele $(T / C)$ & $\chi^{2}$ & $P(\mathrm{df}=1)$ & OR (95\% CI) \\
\hline All CHD cases $(n=263)$ & $77 / 133 / 53$ & & & 0.80 & $287 / 239$ & & & \\
\hline All controls $(n=196)$ & $63 / 98 / 35$ & 0.61 & 0.73 & 0.88 & $224 / 168$ & 0.60 & 0.43 & $0.90(0.69-1.17)$ \\
\hline Female CHD cases $(n=82)$ & $30 / 35 / 17$ & & & 0.26 & $95 / 69$ & & & \\
\hline Female controls $(n=98)$ & $34 / 47 / 17$ & 0.58 & 0.74 & 1.00 & $115 / 81$ & 0.02 & 0.88 & $0.97(0.63-1.47)$ \\
\hline Male CHD cases $(n=181)$ & $47 / 98 / 36$ & & & 0.29 & $192 / 170$ & & & \\
\hline Male controls $(n=98)$ & $29 / 51 / 18$ & 0.43 & 0.80 & 0.68 & $109 / 87$ & 0.33 & 0.56 & $0.90(0.63-1.27)$ \\
\hline
\end{tabular}

analysis of rs7529229 did not reveal significant difference between CHD cases and non-CHD controls $\left(\chi^{2}=0.61, P=\right.$ 0.73). The allelic distribution of rs7529229 did not differ between $\mathrm{CHD}$ cases and non-CHD controls $(P=0.43, \mathrm{OR}=$ $0.90,95 \% \mathrm{CI}=0.69-1.17$, Table 2$)$. We further examined the roles of rs7529229 in males and females separately. However, no significant differences between cases and controls were observed in male and female subgroups (Table 2). In addition, we also performed an age-stratified analysis to investigate whether age influenced the contribution of rs7529229 to the risk of CHD. Again, no significant differences between $\mathrm{CHD}$ cases and controls were observed in all age-stratified subgroups (Table 3).

3.3. Stratified Analyses of rs7529229 between Cases and Controls by Smoking History or Status of Hypertension or Diabetes. Since smoking history, hypertension, and diabetes are risk factors of CAD [21], we further performed stratified association tests by the above three variables. Our results showed that there were no significant differences in the distribution of genotype and allele of rs7529229 between cases and controls (Table 4).

3.4. Association of rs7529229 with the Severity of Coronary Lesions. A linear regression test of the means of Gensini scores with rs7529229 genotype did not show a statistically significant correlation (Table 5). And there was no significant association between Gensini scores and rs7529229 under the dominant $(Z=-0.38, P=0.69$, Table 5$)$ and the recessive models $(Z=-0.50, P=0.61$, Table 5$)$.

3.5. Meta-Analysis of rs7529229 with CHD in Different Populations. A total of 41 studies were selected initially. After reading the full text of these articles, 9 eligible studies were harvested for the current meta-analysis of the association of rs7529229 with CHD [7, 22, 23]. Details of articles in the meta-analysis are shown in Figure 2. Our meta-analysis comprised 11,678 CHD cases and 12,861 controls from two ethnic populations (Europeans and Asians). No significant heterogeneity was found in this meta-analysis $(P=0.441$, $I^{2}=12.5 \%$ ). Our result suggested that rs7529229-C allele was associated with CHD risk. A future subgroup meta-analysis showed that rs7529229 of IL6R gene was a protective factor of CHD, especially in Europeans $(P<0.0001, \mathrm{OR}=0.93$, $95 \%$ CI $=0.89-0.96)$. Sensitivity analyses were repeatedly conducted when each particular study was omitted. As shown in Figure 3, the results were not altered with pooled ORs ranging from 0.92 to 0.94 for the meta-analysis in Europeans and Asians. There was no visual publication bias in Begg's funnel $(P=0.25)$ and Egger's regression plots $(P=0.251$, Figure 4).

\section{Discussion}

In the present study, we aim to replicate previous significant association between IL6R rs7529229 polymorphism and the risk of $\mathrm{CHD}$ in Han Chinese. Our study analyzed the association of rs7529229 with both CHD susceptibility and its severity. We also explored the stratified association of rs7529229 with CHD, though we failed to observe significant associations between IL6R rs7529229 and the risk of CHD. The results of our study were inconsistent with the recent findings from a large study of European samples [7]. We speculated that the discrepancies might be due to ethnic difference in the prevalence of this SNP. In addition, a power calculation showed that our case-control study only had a $12.2 \%$ power to detect a relative risk of rs7529229 at a 
TABLE 3: Post hoc analysis of rs7529229 with the risk of CHD in different age subgroups.

\begin{tabular}{|c|c|c|c|c|c|c|c|c|}
\hline Age group & Genotype (TT/TC/CC) & $\chi^{2}$ & $P(\mathrm{df}=2)$ & HWE & Allele $(T / C)$ & $\chi^{2}$ & $P(\mathrm{df}=1)$ & OR $(95 \% \mathrm{CI})$ \\
\hline$\leq 55 \mathrm{CHD}$ cases $(n=70)$ & $19 / 37 / 14$ & & & 0.80 & $75 / 65$ & & & \\
\hline$\leq 55$ controls $(n=71)$ & $25 / 36 / 10$ & 1.49 & 0.47 & 0.80 & $86 / 56$ & 1.40 & 0.23 & $0.75(0.46-1.20)$ \\
\hline 55-65 CHD cases $(n=95)$ & $23 / 50 / 22$ & & & 0.68 & $96 / 94$ & & & \\
\hline $55-65$ controls $(n=82)$ & $25 / 39 / 18$ & 0.89 & 0.64 & 0.82 & $89 / 75$ & 0.49 & 0.48 & $0.86(0.56-1.30)$ \\
\hline$\geq 65$ CHD cases $(n=98)$ & $35 / 46 / 17$ & & & 0.83 & $116 / 80$ & & & \\
\hline$\geq 65$ controls $(n=43)$ & $13 / 23 / 7$ & 0.54 & 0.76 & 0.75 & $49 / 37$ & 0.12 & 0.72 & $1.09(0.65-1.82)$ \\
\hline
\end{tabular}

TABLE 4: The stratified association analysis of rs7529229.

\begin{tabular}{|c|c|c|c|c|c|c|c|}
\hline Group & Risk factor of CHD & Genotype (TT/TC/CC) & $\chi^{2}$ & $P$ & $T / C$ & $\chi^{2}$ & $P$ \\
\hline $\mathrm{CHD}(n=115)$ & Smoking & $31 / 61 / 23$ & & & $123 / 107$ & & \\
\hline Control $(n=55)$ & Smoking & $15 / 31 / 9$ & 0.339 & 0.844 & $61 / 49$ & 0.117 & 0.732 \\
\hline $\mathrm{CHD}(n=148)$ & No smoking & $46 / 72 / 30$ & & & $164 / 132$ & & \\
\hline Control $(n=141)$ & No smoking & $48 / 67 / 26$ & 0.339 & 0.844 & $163 / 119$ & 0.337 & 0.561 \\
\hline $\mathrm{CHD}(n=165)$ & Hypertension & $48 / 86 / 31$ & & & $182 / 148$ & & \\
\hline Control $(n=102)$ & Hypertension & $36 / 44 / 22$ & 2.061 & 0.375 & $116 / 88$ & 0.15 & 0.699 \\
\hline $\mathrm{CHD}(n=98)$ & No hypertension & $29 / 47 / 22$ & & & $105 / 91$ & & \\
\hline Control $(n=94)$ & No hypertension & $27 / 54 / 13$ & 2.789 & 0.248 & $108 / 80$ & 0.583 & 0.445 \\
\hline $\mathrm{CHD}(n=57)$ & Diabetes & $16 / 27 / 14$ & & & $59 / 55$ & & \\
\hline Control $(n=14)$ & Diabetes & $6 / 5 / 3$ & 1.178 & 0.555 & $17 / 11$ & 0.725 & 0.394 \\
\hline $\mathrm{CHD}(n=206)$ & No diabetes & $61 / 106 / 39$ & & & $228 / 184$ & & \\
\hline Control $(n=182)$ & No diabetes & $57 / 93 / 32$ & 0.191 & 0.909 & $207 / 157$ & 0.183 & 0.669 \\
\hline
\end{tabular}

TABLe 5: Association tests of Gensini scores and CHD.

\begin{tabular}{lcc}
\hline Genotype & Gensini score $($ mean/SD/median $)$ & $F / Z$ \\
\hline TT $(n=77)$ & $56.12 / 56.08 / 35.5$ & \\
TC $(n=133)$ & $48.67 / 43.67 / 33.0$ & \\
CC $(n=53)$ & $46.60 / 43.64 / 36.0$ & 0.30 \\
Recessive model & & \\
TT + TC $(n=210)$ & $51.40 / 48.59 / 35.2$ & 0.85 \\
CC $(n=53)$ & $46.60 / 43.64 / 36.0$ & -0.50 \\
Dominant model & & \\
TC + CC $(n=186)$ & $48.08 / 43.56 / 34.5$ & 0.61 \\
TT $(n=77)$ & $56.12 / 56.08 / 35.5$ & -0.38 \\
\hline
\end{tabular}

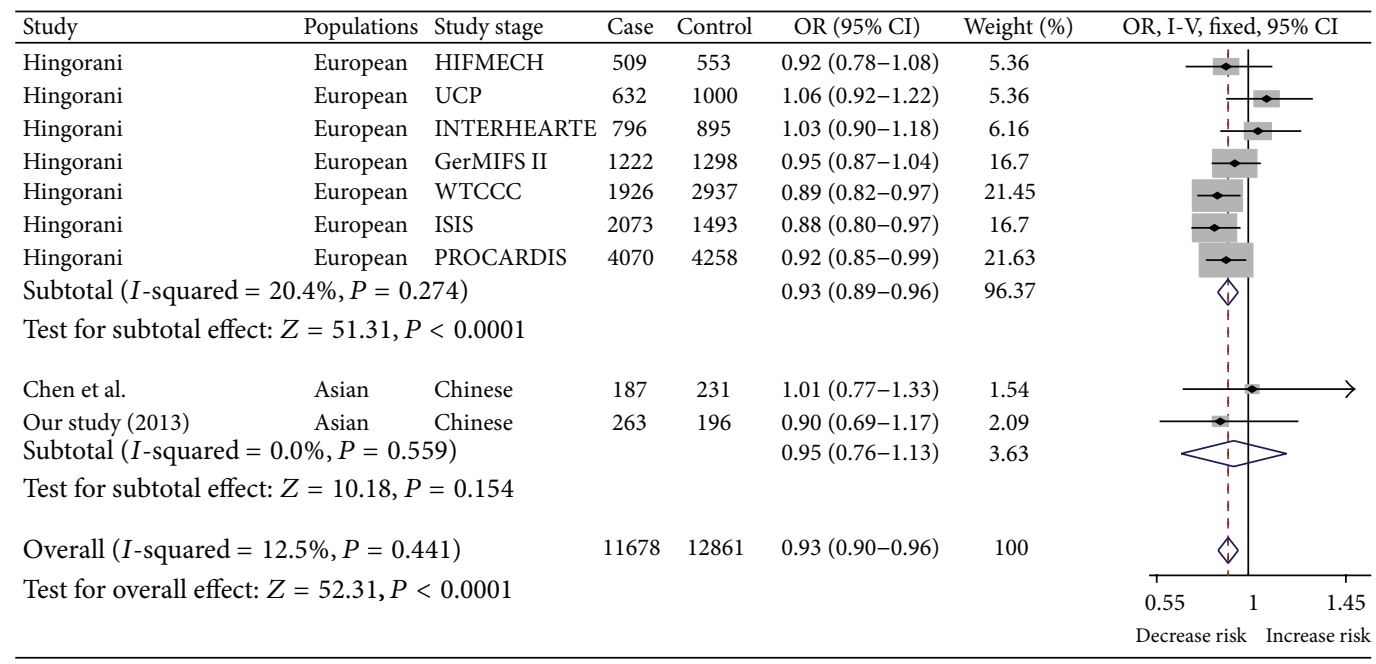

FIGURE 2: Meta-analysis of ten association studies of rs7529229 with CAD. 


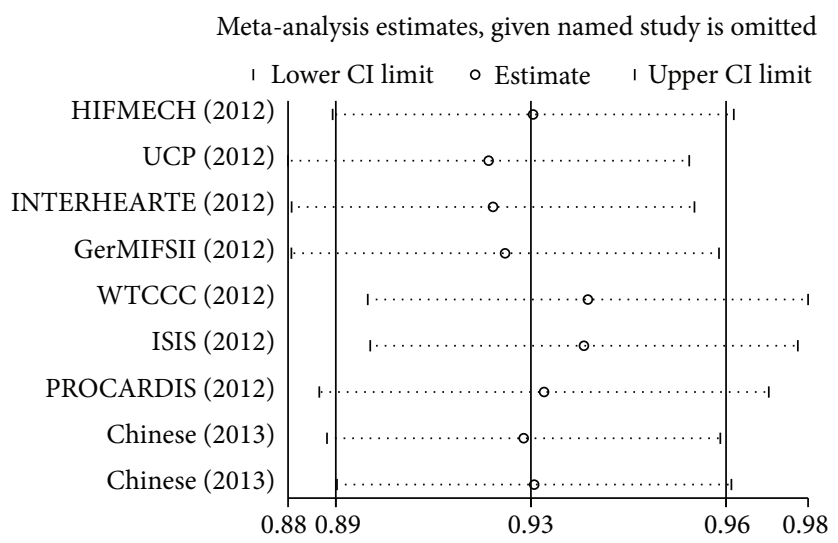

FIGURE 3: Sensitivity analysis for the association between IL6R rs7529229 polymorphism and CHD risk.

Begg's funnel plot with pseudo 95\% confidence limits

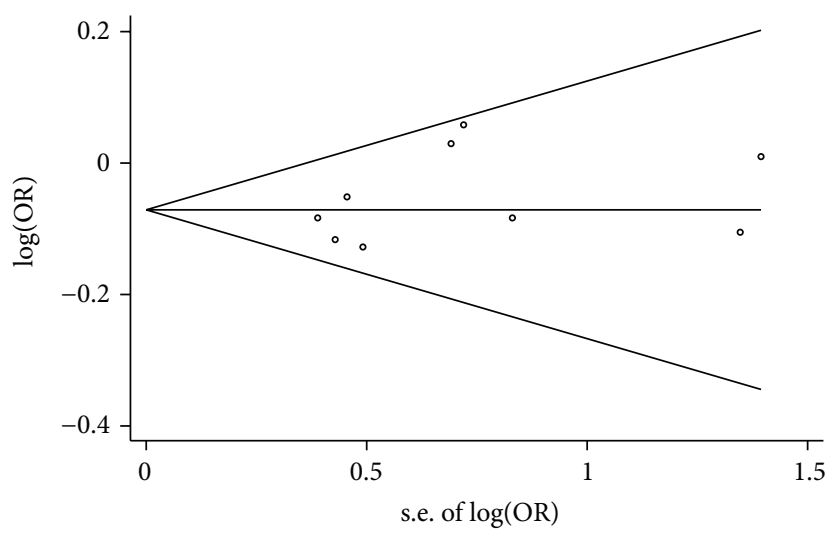

(a)

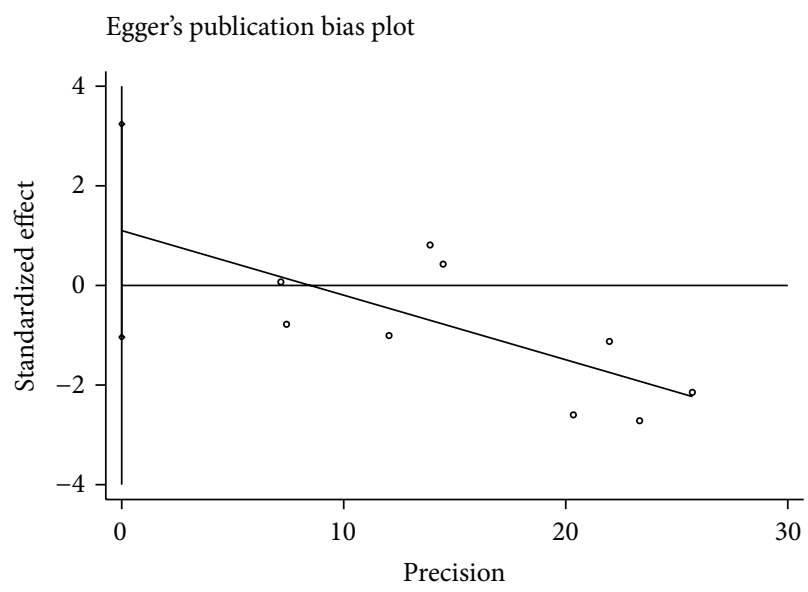

(b)

Figure 4: Begg's funnel plot and Egger's regression plot of 11 association tests between rs7529229 and CHD.

significant level of 0.05 , suggesting that a lack of power was likely to explain our failure to find a significant association.

Our meta-analysis, including a total of 11,678 cases and 12,861 controls, examined the association between the rs7529229 polymorphism and CHD risk. We found that rs7529229 of IL6R gene was associated with the risk of CHD. A further subgroup analysis by race showed that rs7529229 of IL6R gene was a protective factor of CHD, especially in Europeans. Our study is the first association test between rs7629229 and CHD in the Chinese population. We carried out sensitivity analysis to assess the stability of this meta-analysis. Removal of each study did not alter the conclusion of the CHD risk, suggesting the reliability of these results. Meta-analysis can dramatically increase the power of association test through the combination of the data from various studies. For example, the power of some studies in the current meta-analysis is moderate (HIFMECH: 60.1\%; UCP: 55.3\%; INTERHEARTE: 32.2\%; GerMIFSII: 65.2\%; Chen et al.: $11.8 \%)$.

There are several limitations in our study. Firstly, the power of our case-control study only reached $12.2 \%$ at alpha level of 0.05 , so we could not exclude the possibility of lack of power in our study mainly due to the relatively small sample size. Secondly, only one polymorphism of IL6R was investigated in the present study. According to the report in dbSNP, there were at least 256 SNPs on the IL6R gene locus. Therefore, the results of $I L 6 R$ rs7529229 might not stand for the rest of the IL6R SNPs. Thirdly, we only searched the literatures in Chinese for the eligible research included in the meta-analysis. Meanwhile, case-control studies with negative results were more likely to be unpublished. Potential language and publication bias might exist in the meta-analysis.

In conclusion, our meta-analysis has established a strong contribution of rs7529229-C allele to reduced risk of CHD, especially in Europeans, although our case-control study is unable to find association of the IL6R with the risk of CHD. Further investigation on other SNPs on the gene is warranted to validate our findings in the Chinese population.

\section{Conflict of Interests}

The authors declare that there is no conflict of interests regarding the publication of this paper. 


\section{Acknowledgments}

The research was supported by Grants from the National Natural Science Foundation of China (31100919 and 81370207), Advanced Key Scientific and Technological Programs of Ningbo (2011C51001), Fund of Ningbo Science and Technology Innovation team (2011B82015), Natural Science Foundation of the Zhejiang Province (LY13H020008 and LR13H020003), and The Project of Ningbo Medicine and Science (2009A01), K. C. Wong Magna Fund in Ningbo University, and Ningbo Social Development Research Projects (2012C50032). Jianqing Zhou and Xiaoliang Chen are co-first authors of this work.

\section{References}

[1] J. He, D. Gu, X. Wu et al., "Major causes of death among men and women in China," The New England Journal of Medicine, vol. 353, no. 11, pp. 1124-1134, 2005.

[2] R. Ross, "Atherosclerosis-an inflammatory disease," The New England Journal of Medicine, vol. 340, no. 2, pp. 115-126, 1999.

[3] G. K. Hansson, "Inflammation, atherosclerosis, and coronary artery disease," The New England Journal of Medicine, vol. 352, no. 16, pp. 1626-1695, 2005.

[4] O. Harismendy, D. Notani, X. Song et al., "9p21 DNA variants associated with coronary artery disease impair interferon- $\gamma 3$ signalling response," Nature, vol. 470, no. 7333, pp. 264-268, 2011.

[5] T. Naka, N. Nishimoto, and T. Kishimoto, "The paradigm of IL6: from basic science to medicine," Arthritis Research, vol. 4, no. 3, pp. 233-242, 2002.

[6] M. J. Boulanger, D. Chow, E. E. Brevnova, and K. C. Garcia, "Hexameric structure and assembly of the interieukin-6/IL-6 $\alpha$-receptor/gp130 complex," Science, vol. 300, no. 5628, pp. 21012104, 2003.

[7] A. D. Hingorani and J. P. Casas, "The interleukin-6 receptor as a target for prevention of coronary heart disease: a mendelian randomisation analysis," The Lancet, vol. 379, no. 9822, pp. 12141224, 2012.

[8] H. S. Satti, S. Hussain, and Q. Javed, "Association of interleukin6 gene promoter polymorphism with coronary artery disease in pakistani families," The Scientific World Journal, vol. 2013, Article ID 538365, 6 pages, 2013.

[9] Z. C. J. Higgs, D. A. L. Macafee, B. D. Braithwaite, and C. A. Maxwell-Armstrong, "The Seldinger technique: 50 years on," The Lancet, vol. 366, no. 9494, pp. 1407-1409, 2005.

[10] E. Rapaport, R. Bernard, and E. Corday, "Nomenclature and criteria for diagnosis of ischemic heart disease. Report of the Joint International Society and Federation of Cardiology/World Health Organization Task Force on standardization of clinical nomenclature," Circulation, vol. 59, no. 3, pp. 607-609, 1979.

[11] G. G. Gensini, "A more meaningful scoring system for determining the severity of coronary heart disease," The American Journal of Cardiology, vol. 51, no. 3, p. 606, 1983.

[12] J. Wang, K. Chuang, M. Ahluwalia et al., "High-throughput SNP genotyping by single-tube PCR with Tm-shift primers," BioTechniques, vol. 36, no. 6, pp. 885-893, 2005.

[13] S. Germer and R. Higuchi, "Single-tube genotyping without oligonucleotide probes," Genome Research, vol. 9, no. 1, pp. 72$78,1999$.
[14] S. Derzelle, C. Mendy, S. Laroche, and N. Madani, "Use of highresolution melting and melting temperature-shift assays for specific detection and identification of Bacillus anthracis based on single nucleotide discrimination," Journal of Microbiological Methods, vol. 87, no. 2, pp. 195-201, 2011.

[15] S. H. Zhou, M. Liu, W. X. An et al., "Genotyping of human platelet antigen-15 by single closed-tube Tm-shift method," International Journal of Laboratory Hematology, vol. 34, no. 1, pp. 41-46, 2012.

[16] L. Excoffier and H. E. L. Lischer, "Arlequin suite ver 3.5: a new series of programs to perform population genetics analyses under Linux and Windows," Molecular Ecology Resources, vol. 10, no. 3, pp. 564-567, 2010.

[17] W. D. Dupont and W. D. Plummer Jr., "Power and sample size calculations. A review and computer program," Controlled Clinical Trials, vol. 11, no. 2, pp. 116-128, 1990.

[18] D. F. Stroup, J. A. Berlin, S. C. Morton et al., "Meta-analysis of observational studies in epidemiology: a proposal for reporting," Journal of the American Medical Association, vol. 283, no. 15, pp. 2008-2012, 2000.

[19] R. DerSimonian and N. Laird, "Meta-analysis in clinical trials," Controlled Clinical Trials, vol. 7, no. 3, pp. 177-188, 1986.

[20] M. Egger, G. D. Smith, M. Schneider, and C. Minder, "Bias in meta-analysis detected by a simple graphical test," British Medical Journal, vol. 315, no. 7109, pp. 629-634, 1997.

[21] J. Frohlich and A. Al-Sarraf, "Cardiovascular risk and atherosclerosis prevention," Cardiovascular Pathology, vol. 22, no. 1, pp. 16-18, 2013.

[22] P. Elliott, J. C. Chambers, W. Zhang et al., "Genetic loci associated with C-reactive protein levels and risk of coronary heart disease," Journal of the American Medical Association, vol. 302, no. 1, pp. 37-48, 2009.

[23] Z. Chen, Q. Qian, C. Tang, J. Ding, Y. Feng, and G. Ma, "Association of two variants in the interleukin-6 receptor gene and premature coronary heart disease in a Chinese Han population," Molecular Biology Reports, vol. 40, no. 2, pp. 10211026, 2013. 

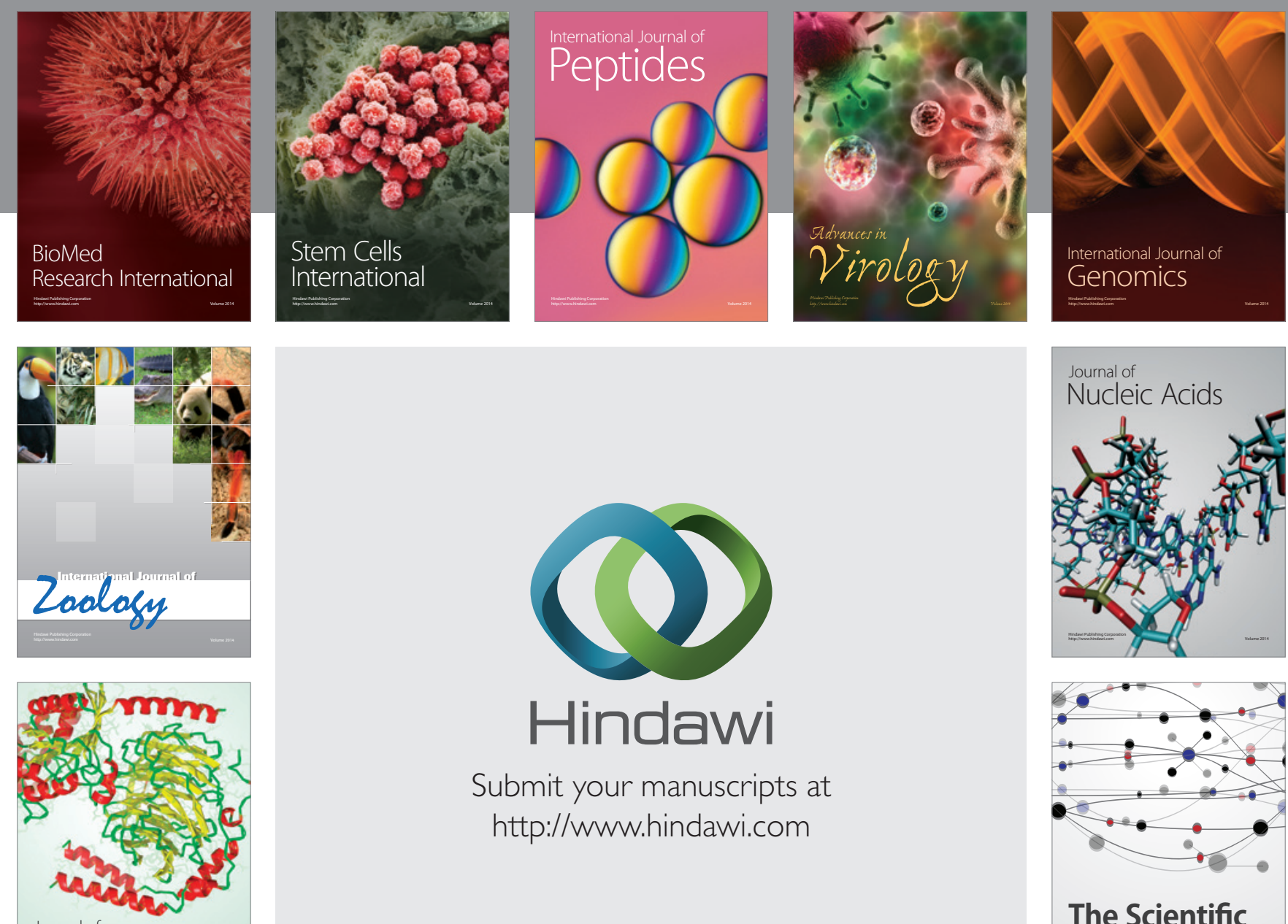

Submit your manuscripts at

http://www.hindawi.com

Journal of
Signal Transduction
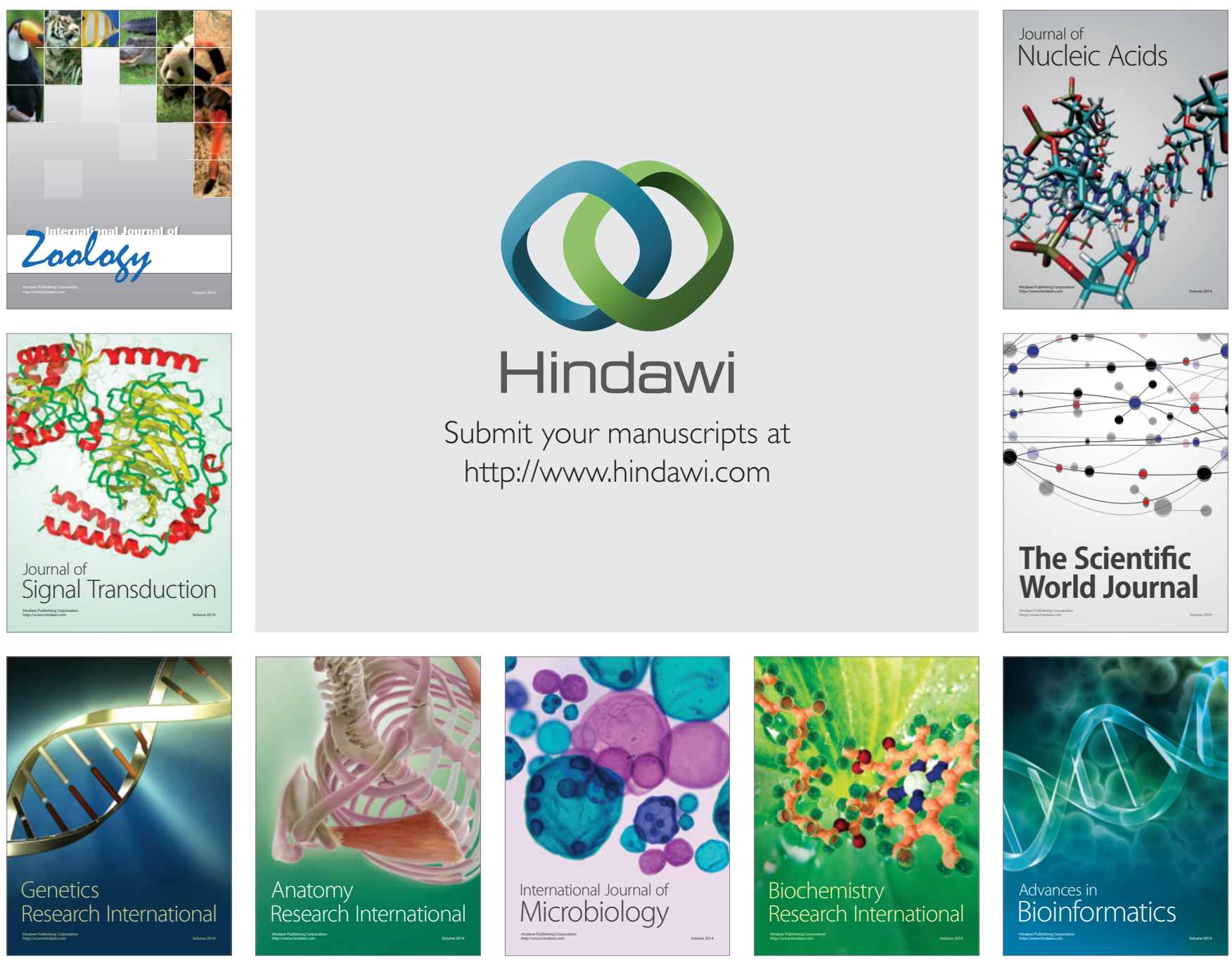

The Scientific World Journal
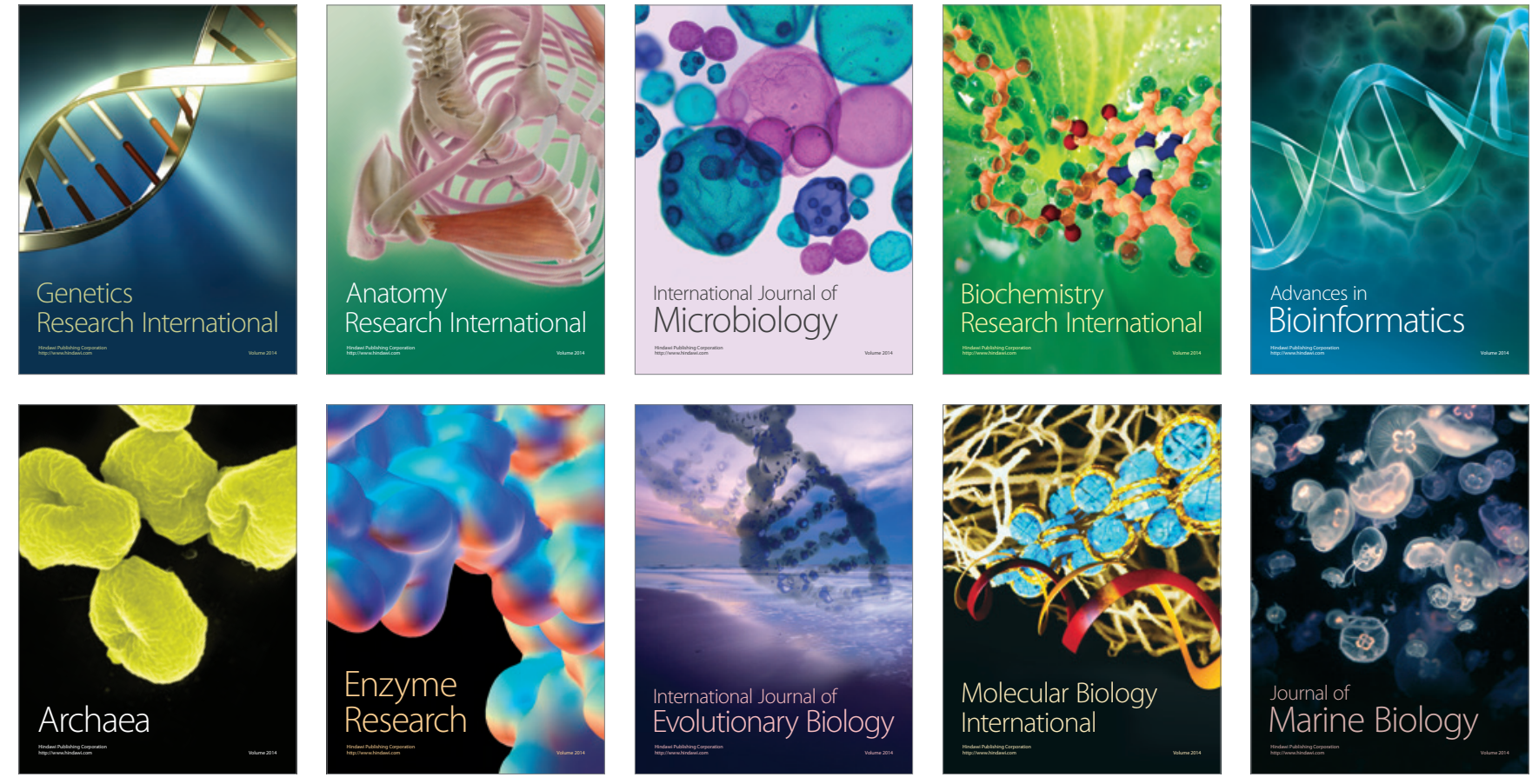